\title{
On $\tau$-supplemented subgroups of finite groups
}

Changwen Li, Xuemei Zhang, and Xiaolan Yi 


\title{
ON $\tau$-SUPPLEMENTED SUBGROUPS OF FINITE GROUPS
}

\author{
CHANGWEN LI, XUEMEI ZHANG, AND XIAOLAN YI
}

Received 30 January, 2013

\begin{abstract}
Let $H$ be a subgroup of a finite group $G$. We say that: (1) $H$ is $\tau$-quasinormal in $G$ if $H$ permutes with every Sylow subgroup $Q$ of $G$ such that $(|H|,|Q|)=1$ and $\left(|H|,\left|Q^{G}\right|\right) \neq 1$; (2) $H$ is $\tau$-supplemented in $G$ if $G$ has a subgroup $T$ of $G$ such that $G=H T$ and $H \cap T \leq H_{\tau G}$, where $H_{\tau G}$ is the subgroup generated by all those subgroups of $H$ which are $\tau$-quasinormal in $G$. We investigate the influence of $\tau$-supplemented subgroups on the structure of finite groups. Some recent known results are generalized and unified.
\end{abstract}

2010 Mathematics Subject Classification: 20D10; 20D20

Keywords: $\tau$-supplemented, $p$-supersolvable supplement, $p$-nilpotent, saturated formation

\section{INTRODUCTION}

This paper deals with finite groups. We use the standard terminology as in [10]. $G$ denotes always a group, $|G|$ is the order of $G$ and the set of distinct primes dividing $|G|$ will be denoted by $\pi(G)$. A group $G$ is called $p$-supersolvable if it is $p$-solvable and all its $G$-chief $p$-factors are cyclic. A group $G$ is called $p$-nilpotent if it is $p$ solvable and all its $G$-chief $p$-factors are central in $G$. Obviously, a $p$-nilpotent group is also a $p$-supersolvable group and $G$ is supersolvable (or nilpotent) if and only if $G$ is $p$-supersolvable (or $p$-nilpotent) for any $p \in \pi(G)$. If $G=H K$ and $K$ is $p$-supersolvable (or supersolvable, $p$-nilpotent), then we call that $H$ has a $p$ supersolvable (or supersolvable, $p$-nilpotent) supplement $K$ in $G$.

A subgroup $H$ of a group $G$ is said to be $S$-quasinormal (or $S$-permutable) in $G$ if $H$ permutes with all Sylow subgroups of $G$, i.e., $H S=S H$ for any Sylow subgroup $S$ of $G$. This concept was first introduced by Kegel in [12]. Later, many authors generalized $S$-quasinormal concept; see, for example, [5, 13, 14, 34]. A subgroup $H$ is said to be $s$-semipermutable in $G$ if $H$ permutes with every Sylow $p$-subgroup of $G$ such that $(p,|H|)=1$. More recently, Lukanenko and Skiba [19] introduced the concept of $\tau$-quasinormal subgroup as follows: A subgroup $H$ of $G$ is said to be $\tau$-quasinormal in $G$ if $H$ permutes with every Sylow subgroup $Q$ of $G$ such that $(|H|,|Q|)=1$ and $\left(|H|,\left|Q^{G}\right|\right) \neq 1$. It is clear that $s$-semipermutability implies $\tau$-quasinormality by definition; however, the converse is not true, as seen in [20, Example 1]. 
On the other hand, some subgroups with supplemented properties were introduced recently. In particular, Ballester-Bolinches, Wang and Guo [7] called that a subgroup $H$ of a group $G$ is $c$-supplemented in $G$ if there is a subgroup $K$ of $G$ such that $G=$ $H K$ and $H \cap K \leq H_{G}$, where $H_{G}$ is the normal core of $H$ in $G$. In 2007, Skiba [25] again gave the concept of $S$-supplemented subgroup as follows: A subgroup $H$ of $G$ is called $S$-supplemented in $G$ if there exists a subgroup $K$ such that $G=H K$ and $H \cap K \leq H_{s}$, where $H_{s G}$ is the subgroup of $H$ generated by all those subgroups of $H$ which are $S$-quasinormal in $G$. If we take $G=<a, b \mid a^{16}=b^{4}=1, b a=$ $a^{3} b>$, then $<b^{2}>$ is an $S$-supplemented subgroup of $G$. However $<b^{2}>$ is not $c$-supplemented in $G$. Hence, $S$-supplemented subgroups generalize $c$-supplemented subgroups.

There is no obvious general relationship between $\tau$-quasinormal subgroups and $S$-supplemented subgroups. Hence it is meaningful to unify and generalize above series subgroups. On the basis of these definitions, we now introduce the following new concept:

Definition 1. A subgroup $H$ of a group $G$ is said to be $\tau$-supplemented in $G$ if $G$ has a subgroup $T$ of $G$ such that $G=H T$ and $H \cap T \leq H_{\tau G}$, where $H_{\tau G}$ is the subgroup generated by all those subgroups of $H$ which are $\tau$-quasinormal in $G$.

The next two examples show that the class of all $\tau$-supplemented subgroups is wider than the class of all $\tau$-quasinormal subgroups and the class of all $S$-supplemented subgroups.

Example 1. Let $G=S_{4}$ be the symmetric group of degree 4 and $H=<(14)>$. Obviously, $H$ is $\tau$-supplemented in $G$. However, $H$ is not $\tau$-quasinormal in $G$.

Example 2. Let $G=<a, b, c \mid a^{5}=b^{4}=c^{5}=1, b^{-1} a b=a^{2},[a, c]=[b, c]=$ $1>$ and $H=<b^{2}>$. It is easy to see that $H$ is $\tau$-supplemented in $G$, but not $S$ supplemented in $G$.

Let $\mathcal{F}$ be a class of groups. We call $\mathscr{F}$ a formation provided that (i) if $G \in \mathscr{F}$ and $H \unlhd G$, then $G / H \in \mathscr{F}$, and (ii) if $G / M$ and $G / N$ are in $\mathscr{F}$, then $G /(M \cap N)$ is in $\mathcal{F}$ for any normal subgroups $M, N$ of $G$. A formation $\mathscr{F}$ is said to be saturated if $G / \Phi(G) \in \mathcal{F}$ implies that $G \in \mathscr{F}$. In this paper, $\mathcal{U}$ and $\mathcal{N}$ will denote the class of all supersolvable groups and the class of all nilpotent groups, respectively. It is well known that both $U$ and $\mathcal{N}$ are saturated formations. A chief factor $H / K$ of a group $G$ is called $\mathscr{F}$-central provided $[H / K]\left(G / C_{G}(H / K)\right) \in \mathscr{F}$. The product of all normal subgroups of $G$ whose $G$-chief factors are $\mathcal{F}$-central in $G$ is called

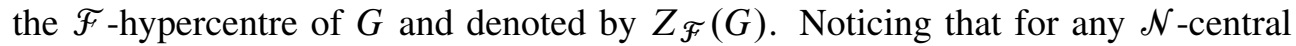
chief factor $H / K$ of $G$ we have $C_{G}(H / K)=G$. Hence the $\mathcal{N}$-hypercentre of $G$ coincides with the hypercentre $Z_{\infty}(G)$ of $G$. Another fact is that any chief factor of $G$ under $Z_{u}(G)$ is of prime order. The major aim of the present paper is to find cyclicity conditions for $G$-chief factors of normal subgroups of a group $G$ by some 
$\tau$-supplemented subgroups. As their applications, we not only extend some known results in $[1,2,4,7,9,15,16,22,28,29,32]$, but also give more simple proofs.

\section{PRELIMINARIES}

Lemma 1 ([19, Lemma 2.3]). Let $G$ be a group and $E \leq K \leq G$.

(1) If $E$ is $\tau$-quasinormal in $G$, then $E$ is $\tau$-quasinormal in $K$.

(2) Suppose that $E$ is normal in $G$ and $\pi(K / E)=\pi(K)$. If $K$ is $\tau$-quasinormal in $G$, then $K / E$ is $\tau$-quasinormal in $G / E$.

(3) Suppose that $E$ is normal in $G$. Then $H E / E$ is $\tau$-quasinormal in $G / E$ fore every $\tau$-quasinormal subgroup $E$ in $G$ satisfying $(|H|,|E|)=1$.

(4) If $E$ is $\tau$-quasinormal in $G$ and $E \leq O_{p}(G)$ for some prime $p$, then $E$ is $S$-quasinormal in $G$.

Lemma 2. Let $H$ be a $\tau$-supplemented subgroup of a group $G$.

(1) If $H \leq L \leq G$, then $H$ is $\tau$-supplemented in $L$.

(2) If $E \unlhd G, E \leq H \leq G$ and $H$ is a p-group for some prime $p$, then $H / E$ is $\tau$-supplemented in $G / E$.

(3) If $H$ is a $\pi$-subgroup and $E$ is a normal $\pi^{\prime}$-subgroup of $G$, then $H E / E$ is $\tau$-supplemented in $G / E$.

Proof. By the hypothesis, there is a subgroup $K$ of $G$ such that $G=H K$ and $H \cap K \leq H_{\tau G}$.

(1) $L=L \cap H K=H(L \cap K)$ and $H \cap(L \cap K)=H \cap K \leq H_{\tau G} \leq H_{\tau K}$. Hence $H$ is $\tau$-supplemented in $L$.

(2) We have $G / E=H K / E=H / E \cdot E K / E$ and $(H / E) \cap(K E / E)=(H \cap$ $K E) / E=(H \cap K) E / E \leq H_{\tau G} E / E=H_{\tau G} / E \leq(H / E)_{\tau(G / E)}$ by Lemma 1. Hence $H / E$ is $\tau$-supplemented in $G / E$.

(3) Since $(|G: K|,|E|)=1$ and $E \unlhd G$, we have $E \leq K$. It is easy to see that $G / E=H E / E \cdot K E / E=H E / E \cdot K / E$ and $(H E / E) \cap(K / E)=(H E \cap K) / E=$ $(H \cap K) E / E \leq H_{\tau G} E / E \leq(H E / E)_{\tau(G / E)}$ by Lemma 1. Hence $H E / E$ is $\tau$ supplemented in $G / E$.

Lemma 3 ([33, p.38, Theorem 7.19]). Let $H$ be a normal subgroup of $G$. Then $H \leq Z_{u}(G)$ if and only if $H / \Phi(H) \leq Z_{u}(G / \Phi(H))$.

Lemma 4 ([26, Lemma A]). Let E be a normal subgroup of a group G. Suppose that for every non-cyclic Sylow subgroup $P$ of $E$, either all maximal subgroups of $P$ or all cyclic subgroups of $P$ of prime order and order 4 are $S$-supplemented in $G$. Then each $G$-chieffactor below $E$ is cyclic.

Lemma 5 ([8, p.362, Proposition 3.11]). If $\mathcal{F}_{1}$ and $\mathcal{F}_{2}$ are two saturated formations such that $\mathcal{F}_{1} \subseteq \mathcal{F}_{2}$, then $Z_{\mathscr{F}_{1}}(G) \leq Z_{\mathcal{F}_{2}}(G)$.

The generalized Fitting subgroup $F^{*}(G)$ of $G$ is the unique maximal normal quasinilpotent subgroup of $G . F^{*}(G)$ is an important subgroup of $G$ and it is a natural 
generalization of $F(G)$. The definition and important properties can be found in [11, Chapter X].

Lemma 6 ([11, X, 13]). Let $G$ be a group. Then:

(1) If $F^{*}(G)$ is solvable, then $F^{*}(G)=F(G)$.

(2) $C_{G}\left(F^{*}(G)\right) \leq F(G)$.

Lemma 7 ([27, Theorem C]). Let $E$ be a normal subgroup of a group $G$. If $F^{*}(E) \leq Z_{U}(G)$, then $E \leq Z_{\mathcal{U}}(G)$.

Lemma 8 ([10, p.434, Satz 5.4 and p.281, Satz 5.2]). If $G$ is a group which is not p-nilpotent but all of its proper subgroups are p-nilpotent, then it is a minimal non-nilpotent group (that is, $G$ is not nilpotent but all of its proper subgroups are nilpotent). Then

(1) $G$ has a normal Sylow $p$-subgroup $P$ for some prime $p$ and $G=P Q$, where $Q$ is a non-normal cyclic $q$-subgroup for some prime $q \neq p$.

(2) $P / \Phi(P)$ is a minimal normal subgroup of $G / \Phi(P)$.

(3) If $P$ is non-abelian and $p>2$, then the exponent of $P$ is $p$; If $P$ is non-abelian and $p=2$, then the exponent of $P$ is 4 .

(4) If $P$ is abelian, then the exponent of $P$ is $p$.

Lemma 9 ([28, Lemma 2.8]). Let $M$ be a maximal subgroup of $G, P$ a normal $p$-subgroup of $G$ such that $G=P M$, where $p$ is a prime. Then $P \cap M$ is a normal subgroup of $G$.

Lemma 10 ([33, p.220, Theorem 6.3]). Let $P$ be a normal p-subgroup of $G$ such that $\left|G / C_{G}(P)\right|$ is a power of $p$. Then $P \leq Z_{\infty}(G)$.

\section{MAIN RESULTS}

Theorem 1. Suppose that $p$ is the smallest prime dividing the order of a group $G$ and $G$ has a normal subgroup $E$ such that $G / E$ is p-nilpotent. If every cyclic subgroup $H$ of $E$ with prime order $p$ or order 4 (if $p=2$ ) having no $p$-supersoluble supplement in $G$ is $\tau$-supplemented in $G$, then $G$ is p-nilpotent.

Proof. Suppose that the theorem is false, and let $G$ be a counterexample of minimal order .

(1) The hypotheses are inherited by all proper subgroups of $G$ and $G$ is a group which is not nilpotent but whose proper subgroups are all nilpotent.

In fact, $\forall K<G$, since $G / E$ is $p$-nilpotent, $K /(K \cap E) \cong K E / E$ is also $p$ nilpotent. Let $H$ be any cyclic subgroup of $K \cap E$ with prime order $p$ or order 4 (if $p=2$ ). Obviously, $H$ is a cyclic subgroup of $E$ with prime order $p$ or order 4. If $H$ has a $p$-supersoluble supplement $T$ in $G$, then $H$ has a $p$-supersoluble supplement $T \cap K$ in $K$. If $H$ is $\tau$-supplemented in $G$, then $H$ is $\tau$-supplemented in $K$ by Lemma 
2(1). Thus $K$ satisfies the hypotheses of the theorem. By the choice of $G, K$ is $p$ nilpotent. Then $G$ is a group which is not $p$-nilpotent but whose proper subgroups are all $p$-nilpotent. By Lemma $8, G=P Q$ and $P \unlhd G$.

(2) $G /(P \cap E)$ is $p$-nilpotent.

Since $G / P \cong Q$ is $p$-nilpotent, $G / E$ is $p$-nilpotent and $G /(P \cap E) \lesssim G / P \times$ $G / E$, we have $G /(P \cap E)$ is $p$-nilpotent.

(3) $P \leq E$.

If $P \not \leq E$, then $P \cap E<P$. So $Q(P \cap E)<Q P=G$. Thus $Q(P \cap E)$ is nilpotent by Step (1) and so $Q(P \cap E)=Q \times(P \cap E)$. Since $G /(P \cap E)=P /(P \cap E)$. $Q(P \cap E) /(P \cap E)$, it follows that $Q(P \cap E) /(P \cap E) \unlhd G /(P \cap E)$ by Step (2). Now $Q$ char $Q(P \cap E) \unlhd G$ implies that $G=P \times Q$, a contradiction.

(4) For every cyclic subgroup $L$ of $P$ with order $p$ or 4 , if there is a subgroup $T$ of $G$ such that $G=L T$, then $T=G$.

Let $L$ be a cyclic subgroup of prime order $p$ or of order 4 in $P$ and assume that there exists a subgroup $T$ of $G$ such that $G=L T$. Obviously, $P=P \cap G=$ $P \cap L T=L(P \cap T)$. Since $P / \Phi(P)$ is abelian, we have $(P \cap T) \Phi(P) / \Phi(P) \unlhd$ $G / \Phi(P)$. By Step (1), $P \cap T \leq \Phi(P)$ or $P=(P \cap T) \Phi(P)=P \cap T$. If the former holds, then $L=P$ and so $G$ is $p$-nilpotent by [24, P.280, Theorem 10.1.9], a contradiction. Hence $P=P \cap T$ and $T=G$.

(5) Every cyclic subgroup $L$ of $P$ with prime order $p$ or of order 4 is $S$-quasinormal in $G$.

If $L$ has a $p$-supersoluble supplement $T$ in $G$, then $G=T$ is $p$-supersoluble by Step (4) and so $G$ is $p$-nilpotent since $p$ is the smallest prime dividing $|G|$, a contradiction. Thus we may assume all cyclic subgroups of $P$ with order $p$ or 4 are $\tau$-supplemented in $G$. In view of Step (4), all cyclic subgroups of $P$ with order $p$ or 4 are $\tau$-quasinormal in $G$. By Lemma 1(4), all cyclic subgroups of $P$ with order $p$ or 4 are $S$-quasinormal in $G$.

(6) Final contradiction.

For every $x \in P$, we have $|\langle x\rangle|=p$ or 4 by Step (1), and so $\langle x\rangle$ is $S$ quasinormal in $G$ by Step (5). By [24, P.280, Theorem 10.1.9], we have $\langle x>Q$ is a proper subgroup of $G$, and so $\langle x\rangle Q=<x>\times Q$ by Step (1). Then we conclude that $G=P \times Q$, a contradiction.

Theorem 2. Suppose that $P$ is a normal $p$-subgroup of a group $G$. If every cyclic subgroup of $P$ with order $p$ or 4 (if $p=2$ ) having no p-supersoluble supplement in $G$ is $\tau$-supplemented in $G$, then $P \leq Z_{U}(G)$.

Proof. We distinguish two cases:

Case I. $p=2$.

Pick an arbitrary Sylow $q$-subgroup $G_{q}$ of $G$, where $q \neq 2$. Consider the subgroup $W=G_{q} P$. Let $L$ be a cyclic subgroup of $P$ with order 2 or 4 . If $L$ has a 2supersoluble supplement $T$ in $G$, then $L$ has a 2-supersoluble supplement $T \cap W$ in $W$. If $L$ is $\tau$-supplemented in $G$, then $L$ is also $\tau$-supplemented in $W$ by Lemma 
2(1). Applying Theorem 1, we have $W$ is 2-nilpotent. Hence $W=P \times G_{q}$. This implies that $O^{p}(G) \leq C_{G}(P)$. In view of Lemma $10, P \leq Z_{\infty}(G)$. Consequently, $P \leq Z_{U}(G)$ from Lemma 5.

Case II. $p>2$.

First suppose that some cyclic subgroup $L$ of $P$ with order $p$ has a $p$-supersoluble supplement $T$ in $G$. So $G=L T=P T$. If $T=G$, then $P \leq Z_{u}(G)$ and we are done. Hence we may assume that $T<G$, which shows that $L \cap T=1$ and $T$ is a maximal subgroup of $G$. Clearly, $P=P \cap L T=L(P \cap T)$ and $P \cap T$ is a maximal subgroup of $P$. By Lemma $9, P \cap T$ is normal in $G$. Since every cyclic subgroup of $P \cap T$ with order $p$ having no $p$-supersoluble supplement in $G$ is $\tau$-supplemented in $G, P \cap T \leq Z_{u}(G)$ by induction. Noticing that $P /(P \cap T)$ is a normal subgroup of $G /(P \cap T)$ with order $p$, we have $P /(P \cap T) \leq Z_{u}(G / P \cap T)$. It follows that $P \leq Z_{u}(G)$. Now we may assume that all cyclic subgroups of $P$ with order $p$ are $\tau$-supplemented in $G$. From Lemma 1(4) we have that all cyclic subgroups of $P$ with order $p$ are $S$-supplemented in $G$ since $P \leq O_{p}(G)$. In view of Lemma 4, we have also $P \leq Z_{U}(G)$.

Theorem 3. Let $E$ be a normal subgroup of a group $G$. Suppose that for each $p \in$ $\pi(E)$ and non-cyclic Sylow p-subgroup $P$ of $E$, all cyclic subgroups of $P$ with order $p$ or 4 (if $p=2$ ) having no $p$-supersoluble supplement in $G$ are $S$-supplemented in $G$. Then $E \leq Z_{u}(G)$.

Proof. Let $q$ be the smallest prime dividing $|E|$ and $L$ a cyclic subgroup of the Sylow $q$-subgroup $Q$ of $E$ with order $q$ or 4 (if $q=2$ ). If $L$ has a $q$-supersoluble supplement $T$ in $G$, then $L$ has a $q$-supersoluble supplement $T \cap E$ in $E$. If $L$ is $\tau$ supplemented in $G$, then $L$ is also $\tau$-supplemented in $E$ by Lemma 2(1). In view of Theorem 1, $E$ is $q$-nilpotent. Let $E_{q^{\prime}}$ be the normal $q^{\prime}$-complement of $E$. If $E=Q$, then $E \leq Z_{U}(G)$ by Theorem 2. Hence we may assume that $E_{q^{\prime}} \neq 1$. Since $E_{q^{\prime}}$ char $E \unlhd G, E_{q^{\prime}} \unlhd G$. By the hypothesis of the theorem, for each $p \in \pi\left(E_{q^{\prime}}\right)$ and noncyclic Sylow $p$-subgroup $P$ of $E_{q^{\prime}}$, all cyclic subgroups of $P$ with order $p$ having no $p$-supersoluble supplement in $G$ are $S$-supplemented in $G$. By induction, $E_{q^{\prime}} \leq$ $Z_{u}(G)$. By Lemma 2(3), it is easy to see that all cyclic subgroups of $Q E_{q^{\prime}} / E_{q^{\prime}}$ with order $q$ or 4 (if $q=2$ ) having no $q$-supersoluble supplement in $G / E_{q^{\prime}}$ are $S$-supplemented in $G / E_{q^{\prime}}$. By induction, we have also $E / E_{q^{\prime}} \leq Z_{U}\left(G / E_{q^{\prime}}\right)$. It follows that $E \leq Z_{u}(G)$.

Corollary 1. Let E be a normal subgroup of a group $G$. Suppose that every cyclic subgroup of each non-cyclic Sylow subgroup of $E$ with prime order or order 4 having no supersoluble supplement in $G$ are $S$-supplemented in $G$. Then $E \leq Z_{u}(G)$.

Theorem 4. Let $P$ be a normal p-subgroup of a group $G$, where $p$ is a prime dividing the order of $G$. Suppose that every maximal subgroup of $P$ having no $p$ supersoluble supplement in $G$ is $\tau$-supplemented in $G$. Then $P \leq Z_{u}(G)$. 
Proof. We distinguish two cases:

Case I. $\Phi(P) \neq 1$.

Obviously, $P / \Phi(P)$ is a normal $p$-subgroup of $G / \Phi(P)$. Let $P_{1} / \Phi(P)$ be a maximal subgroup of $P / \Phi(P)$. Then $P_{1}$ is a maximal subgroup of $P$. If $P_{1}$ has a $p$-supersoluble supplement $T$ in $G$, then $P_{1} / \Phi(P)$ has a $p$-supersoluble supplement $T \Phi(P) / \Phi(P)$ in $G / \Phi(P)$. If $P_{1}$ is $\tau$-supplemented in $G$, then $P_{1} / \Phi(P)$ is $\tau$ supplemented in $G / \Phi(P)$ by Lemma 2. Therefore, $G / \Phi(P)$ satisfies the hypothesis of the theorem. By induction, $P / \Phi(P) \leq Z_{u}(G / \Phi(P))$. In view of Lemma 3, we have $P \leq Z_{u}(G)$.

Case II. $\Phi(P)=1$.

This shows that $P$ is abelian. First suppose that some maximal subgroup $V$ of $P$ has a $p$-supersoluble supplement $T$ in $G$. Then $G=V T=P T$ and $P \cap T \neq$ 1. Since $P \cap T \unlhd T$, we may assume that $T$ has a minimal normal subgroup $N$ contained in $P \cap T$. It is clear that $|N|=p$. From $G=P T$, we have $N$ is also normal in $G$. With the similar argument in Case I, the hypothesis of the theorem holds for $(G / N, P / N)$. By induction, we have $P / N \leq Z_{u}(G / N)$. It follows that $P \leq$ $Z_{u}(G)$. Now we may assume that every maximal subgroup of $P$ is $\tau$-supplemented in $G$. In view of Lemma 1, every maximal subgroup of $P$ is $S$-supplemented in $G$ since $P \leq O_{p}(G)$. Then we have also $P \leq Z_{u}(G)$ by Lemma 4.

Corollary 2. Let $P$ be a normal p-subgroup of $G$. Suppose that every maximal subgroup of $P$ having no supersoluble supplement in $G$ is $\tau$-supplemented in $G$. Then $P \leq Z_{u}(G)$.

In connection with Theorem 3 and Lemma 4 the following natural question arises:

Question. Let $E$ be a normal subgroup of a group $G$. Suppose that for every noncyclic Sylow subgroup $P$ of $E$ all maximal subgroups of $P$ having no supersoluble supplement in $G$ are $\tau$-supplemented in $G$. Is then $E \leq Z_{U}(G)$ ?

\section{Some Applications}

Theorem 5. Let $\mathcal{F}$ be a saturated formation containing $\mathcal{U}$. Suppose that $G$ is a group with a solvable normal subgroup $E$ such that $G / E \in \mathcal{F}$. If every maximal subgroup of each non-cyclic Sylow subgroup of $F(E)$ having no supersoluble supplement in $G$ is $\tau$-supplemented in $G$, then $G \in \mathcal{F}$.

Proof. By Theorem 4, $F(E) \leq Z_{\mathcal{U}}(G)$. Since $U \subseteq \mathcal{F}$, we have that $F(E) \leq$ $Z_{\mathcal{F}}(G)$ by Lemma 5. In view of the solvability of $E$ and Lemma $6, F^{*}(E)=$ $F(E) \leq Z_{\mathcal{F}}(G)$. By Lemma $7, E \leq Z_{\mathcal{F}}(G)$. Since $G / Z_{\mathcal{F}}(G) \cong$ $(G / E) /\left(Z_{\mathscr{F}}(G) / E\right) \in \mathscr{F}$, we have $G \in \mathscr{F}$.

Theorem 6. Let $\mathcal{F}$ be a saturated formation containing $U$. If there is a normal subgroup $E$ of a group $G$ such that $G / E \in \mathcal{F}$ and every cyclic subgroup of $E$ with 
prime order or order 4 having no supersoluble supplement in $G$ is $\tau$-supplemented in $G$, then $G \in \mathcal{F}$.

Proof. Since $E \leq Z_{U}(G)$ by Theorem 3 and $Z_{U}(G) \leq Z_{\mathscr{F}}(G)$ by Lemma 5, we have $E \leq Z_{\mathscr{F}}(G)$. Hence $G / Z_{\mathscr{F}}(G) \cong(G / E) /\left(Z_{\mathscr{F}}(G) / E\right) \in \mathcal{F}$. It follows that $G \in \mathcal{F}$.

Theorem 7. Let $\mathcal{F}$ be a saturated formation containing $U$ and let $G$ be a group. If there is a normal subgroup $E$ such that $G / E \in \mathcal{F}$ and every cyclic subgroup of $F^{*}(E)$ with prime order or order 4 having no supersoluble supplement in $G$ is $\tau$ supplemented in $G$, then $G \in \mathcal{F}$.

Proof. By Theorem $3, F^{*}(E) \leq Z_{U}(G)$ and since $Z_{U}(G) \leq Z_{\mathcal{F}}(G)$ by Lemma 5, we have $F^{*}(E) \leq Z_{\mathscr{F}}(G)$. In view of Lemma $7, E \leq Z_{\mathscr{F}}(G)$. Hence $G \in \mathcal{F}$ since $G / E \in \mathcal{F}$.

Theorem 8. Let $\mathcal{F}$ be a saturated formation containing $\mathcal{U}$. Suppose that $G$ is a group with a solvable normal subgroup $E$ such that $G / E \in \mathcal{F}$. If every cyclic subgroup of $F(E)$ with prime order or order 4 having no supersoluble supplement in $G$ is $\tau$-supplemented in $G$, then $G \in \mathcal{F}$.

Proof. Since $E$ is solvable, $F^{*}(E)=F(E)$ by Lemma 6. Applying Theorem 7, we have $G \in \mathcal{F}$.

Corollary 3 ([22, Theorem 3.1]). Assume that $G$ is solvable and every maximal subgroup of the Sylow subgroups of $F(G)$ is normal in $G$. Then $G$ is supersolvable.

Corollary 4 ([4, Corollary 4.4]). Suppose that $G$ is a solvable group with a normal subgroup $E$ such that $G / E$ is supersolvable. If all maximal subgroups of any Sylow subgroup of $F(E)$ are $S$-quasinormal in $G$, then $G$ is supersolvable.

A subgroup $H$ of a group $G$ is $c$-normal in $G$ if there is a normal subgroup $K$ of $G$ such that $G=H K$ and $H \cap K \leq H_{G}$, where $H_{G}$ is the normal core of $H$ in $G$.

Corollary 5 ([15, Theorem 2]). Let $G$ be a group and $E$ a solvable normal subgroup of $G$ such that $G / E$ is supersolvable. If every maximal subgroup of each Sylow subgroup of $F(E)$ is c-normal in $G$, then $G$ is supersolvable.

Corollary 6 ([34, Theorem 2]). Let $\mathcal{F}$ be a saturated formation containing $U$, the class of all supersolvable groups. Suppose that $G$ is a group with a solvable normal subgroup $E$ such that $G / E \in \mathcal{F}$. If all maximal subgroups of all Sylow subgroups of $F(E)$ are s-semipermutable in $G$, then $G \in \mathcal{F}$.

Corollary 7 ([16, Theorem 1.2]). Suppose that $G$ is a solvable group with a normal subgroup $E$ such that $G / E$ is supersolvable. If every maximal subgroup of each Sylow subgroup of $F(E)$ is complement in $G$, then $G$ is supersolvable. 
Corollary 8 ([30, Theorem 1]). Let $\mathcal{F}$ be a saturated formation containing $\mathcal{U}$. Suppose that $G$ is a group with a solvable normal subgroup $E$ such that $G / E \in \mathcal{F}$. If every maximal subgroup of each Sylow subgroup of $F(E)$ is c-normal in $G$, then $G \in \mathcal{F}$.

Corollary 9 ([1, Theorem 1.4]). Let $\mathcal{F}$ be a saturated formation containing $\mathcal{U}$. Suppose that $G$ is a solvable group with a normal subgroup $E$ such that $G / E \in \mathcal{F}$. If every maximal subgroup of each Sylow subgroup of $F(E)$ is $S$-quasinormal in $G$, then $G \in \mathcal{F}$.

Corollary 10 ([28, Theorem 4.5]). Let $\mathcal{F}$ be a saturated formation containing $\mathcal{U}$. Suppose that $G$ is a group with a solvable normal subgroup $E$ such that $G / E \in \mathcal{F}$. If every maximal subgroup of each Sylow subgroup of $F(E)$ is c-supplemented in $G$, then $G \in \mathcal{F}$.

Corollary 11 ([9, Theorem 1.6]). Let $\mathcal{F}$ be a saturated formation containing $\mathcal{U}$. Suppose that $G$ is a group with a solvable normal subgroup $E$ such that $G / E \in \mathcal{F}$. If every maximal subgroup of each Sylow subgroup of $F(E)$ is complemented in $G$, then $G \in \mathcal{F}$.

A subgroup $H$ is called $Q$-supplemented in a group $G$, if there exists a subgroup $K$ of $G$ such that $G=H K$ and $H \cap K$ is contained in $H_{Q G}$, where $H_{Q G}$ is the maximal quasinormal subgroup of $G$ contained in $H$.

Corollary 12 ([21, Theorem 3.6]). Let $\mathcal{F}$ be a saturated formation containing $\mathcal{U}$. Suppose that $G$ is a group with a solvable normal subgroup $E$ such that $G / E \in \mathcal{F}$. If every maximal subgroup of each Sylow subgroup of $F(E)$ is $Q$-supplemented in $G$, then $G \in \mathcal{F}$.

Corollary 13 ([34, Theorem 3]). Let $\mathcal{F}$ be a saturated formation containing $\mathcal{U}$. If there is a normal subgroup $E$ of $G$ such that $G / E \in \mathcal{F}$ and every cyclic subgroup of $E$ with prime order or order 4 is s-semipermutable in $G$, then $G \in \mathcal{F}$.

Corollary 14 ([29, Theorem 4.2]). If every cyclic subgroup of a group $G$ with prime order or order 4 is c-normal in $G$, then $G$ is supersolvable.

Corollary 15 ([35, Theorem 3.1]). Let $G$ be a group and $E$ a normal subgroup of a group $G$ such that $G / E$ is supersolvable. If every minimal subgroup of $E$ is $c$-supplemented in $G$ and if every cyclic subgroup of $E$ with order 4 is $c$-normal in $G$, then $G$ is supersolvable.

Corollary 16 ([7, Theorem 4.1]). If every cyclic subgroup of $G^{u}$ with prime order or order 4 is c-supplemented in $G$, then $G$ is supersolvable.

Corollary 17 ([23, Theorem 3.9]). Let $\mathcal{F}$ be a saturated formation containing $\mathcal{U}$. Then $G \in \mathcal{F}$ if and only if there is a normal subgroup $E$ of $G$ such that $G / E \in \mathscr{F}$ and the subgroups prime order or order 4 of $E$ with are c-normal in $G$. 
Corollary 18 ([2, Theorem 1]). Let $\mathcal{F}$ be a saturated formation containing $\mathcal{U}$. If there is a normal subgroup $E$ of $G$ such that $G / E \in \mathcal{F}$ and every cyclic subgroup of $E$ with prime order or order 4 is $S$-quasinormal in $G$, then $G \in \mathcal{F}$.

Corollary 19 ([6, Theorem 3.4]). Let $\mathcal{F}$ be a saturated formation containing $\mathcal{U}$. If every cyclic subgroup of $G^{\mathscr{F}}$ with prime order or order 4 is $c$-normal in $G$, then $G \in \mathcal{F}$.

Corollary 20 ([31, Theorem 3.2]). Let $\mathcal{F}$ be a saturated formation containing $\mathcal{U}$ and let $G$ be a group. If there is a normal subgroup $E$ such that $G / E \in \mathcal{F}$ and the subgroups of $F^{*}(E)$ with prime order or order 4 are c-normal in $G$, then $G \in \mathcal{F}$.

Corollary 21 ([18, Theorem 3.3]). Let $\mathcal{F}$ be a saturated formation containing $U$ and let $G$ be a group. If there is a normal subgroup $E$ such that $G / E \in \mathcal{F}$ and the subgroups of $F^{*}(E)$ with prime order or order 4 are $S$-quasinormal in $G$, then $G \in \mathcal{F}$.

Corollary 22 ([32, Theorem 1.2]). Let $\mathcal{F}$ be a saturated formation containing $U$ and let $G$ be a group. If there is a normal subgroup $E$ such that $G / E \in \mathscr{F}$ and the subgroups of $F^{*}(E)$ with prime order or order 4 are c-supplemented in $G$, then $G \in \mathcal{F}$.

Corollary 23 ([3, Corollary 1]). Suppose that $G$ is a group with a normal solvable subgroup $E$ such that $G / E$ is supersolvable. If every subgroup of $F(E)$ of prime order or order 4 is $S$-quasinormal in $G$, then $G$ is supersolvable.

Corollary 24 ([15, Theorem 3]). Let $G$ be a group and $E$ a solvable normal subgroup of $G$ such that $G / E$ is supersolvable. If all minimal subgroups and all cyclic subgroups of $F(E)$ with order 4 are c-normal in $G$, then $G$ is supersolvable.

Corollary 25 ([28, Theorem 4.1]). Let $\mathcal{F}$ be a saturated formation containing $U$. Suppose that $G$ is a group with a solvable normal subgroup $E$ such that $G / E \in$ $\mathcal{F}$. If all minimal subgroups and all cyclic subgroups of $F(E)$ with order 4 is $c$ supplemented in $G$, then $G \in \mathcal{F}$.

Corollary 26 ([30, Theorem 2]). Let $\mathcal{F}$ be a saturated formation containing $U$. Suppose that $G$ is a group with a solvable normal subgroup $E$ such that $G / E \in \mathcal{F}$. If all minimal subgroups and all cyclic subgroups of $F(E)$ with order 4 is c-normal in $G$, then $G \in \mathcal{F}$.

Corollary 27 ([17, Theorem 3]). Let $\mathcal{F}$ be a saturated formation containing $\mathcal{U}$. A group $G \in \mathcal{F}$ if and only if there is a solvable normal subgroup $E$ of $G$ such that $G / E \in \mathcal{F}$ and the subgroups of $F(E)$ with prime order or order 4 is c-normal in $G$.

Corollary 28 ([3, Theorem ]). A group $G \in \mathcal{F}$ if and only if there is a solvable normal subgroup $E$ of $G$ such that $G / E \in \mathcal{F}$ and the subgroups of $F(E)$ with prime order or order 4 is $S$-quasinormal in $G$. 
Corollary 29 ([34, Theorem 4]). Let $\mathcal{F}$ be a saturated formation containing $\mathcal{U}$. Suppose that $G$ is a group with a solvable normal subgroup $E$ such that $G / E \in$ $\mathcal{F}$. If all minimal subgroups and all cyclic subgroups of $F(E)$ with order 4 is $s$ semipermutable in $G$, then $G \in \mathscr{F}$.

Acknowledgement. The authors would like to thank the referee who read the manuscript carefully and contributed a lot of valuable suggestions and useful comments. This research was partly supported by the Natural Science Foundation of China (grant no.11101369) and the Priority Academic Program Development of Jiangsu Higher Education Institutions.

\section{REFERENCES}

[1] M. Asaad, "On maximal subgroups of Sylow subgroups of finite groups," Commun. Algebra, vol. 26, no. 11, pp. 3647-3652, 1998.

[2] M. Asaad, A. Ballester-Bolinches, and M. C. Pedraza Aguilera, "A note on minimal subgroups of finite groups," Commun. Algebra, vol. 24, no. 8, pp. 2771-2776, 1996.

[3] M. Asaad and P. Csörgö, "The influence of minimal subgroups on the structure of finite groups," Arch. Math., vol. 72, no. 6, pp. 401-404, 1999.

[4] M. Asaad, M. Ramadan, and A. Shaalan, "Influence of $\pi$-quasinormality on maximal subgroups of Sylow subgroups of Fitting subgroup of a finite group," Arch. Math., vol. 56, no. 6, pp. 521-527, 1991.

[5] A. Ballester-Bolinches and M. C. Pedraza-Aguilera, "Sufficient conditions for supersolubility of finite groups," J. Pure Appl. Algebra, vol. 127, no. 2, pp. 113-118, 1998.

[6] A. Ballester-Bolinches and Y. Wang, "Finite groups with some $c$-normal minimal subgroups," $J$. Pure Appl. Algebra, vol. 153, no. 2, pp. 121-127, 2000.

[7] A. Ballester-Bolinches, Y. Wang, and X. Guo, " $c$-supplemented subgroups of finite groups," Glasg. Math. J., vol. 42, no. 3, pp. 383-389, 2000.

[8] K. Doerk and T. Hawkes, Finite soluble groups, ser. De Gruyter Expositions in Mathematics. Berlin: W. de Gruyter, 1992, vol. 4.

[9] X. Guo and K. P. Shum, "Complementarity of subgroups and the structure of finite groups," $A l$ gebra Colloq., vol. 13, no. 1, pp. 9-16, 2006.

[10] B. Huppert, Endliche Gruppen. I., ser. Die Grundlehren der mathematischen Wissenschaften in Einzeldarstellungen. Berlin-Heidelberg-New York: Springer-Verlag, 1967, vol. 134.

[11] B. Huppert and N. Blackburn, Finite groups. III, ser. Grundlehren der Mathematischen Wissenschaften. Berlin-Heidelberg-New York: Springer-Verlag, 1982, vol. 243.

[12] O. H. Kegel, "Sylow-Gruppen und Subnormalteiler endlicher Gruppen," Math. Z., vol. 78, pp. 205-221, 1962.

[13] C. Li, "Finite groups with some primary subgroups ss-quasinormally embedded," Indian J. Pure Appl. Math., vol. 42, no. 5, pp. 291-306, 2012.

[14] C. Li, "On weakly $s s$-permutable subgroups of finite groups," Math. Notes, Miskolc, vol. 12, no. 2, pp. 201-208, 2011.

[15] D. Li and X. Guo, "The influence of $c$-normality of subgroups on the structure of finite groups. II," Commun. Algebra, vol. 26, no. 6, pp. 1913-1922, 1998.

[16] D. Li and X. Guo, "On complemented subgroups of finite groups," Chin. Ann. Math., Ser. B, vol. 22, no. 2, pp. 249-254, 2001.

[17] Y. Li, "Some notes on the minimal subgroups of Fitting subgroups of finite groups," J. Pure Appl. Algebra, vol. 171, no. 2-3, pp. 289-294, 2002. 
[18] Y. Li and Y. Wang, "The influence of minimal subgroups on the structure of a finite group," Proc. Am. Math. Soc., vol. 131, no. 2, pp. 337-341, 2003.

[19] V. O. Lukyanenko and A. N. Skiba, "On weakly $\tau$-quasinormal subgroups of finite groups," Acta Math. Hung., vol. 125, no. 3, pp. 237-248, 2009.

[20] V. O. Lukyanenko and A. N. Skiba, "Finite groups in which $\tau$-quasinormality is a transitive relation," Rend. Semin. Mat. Univ. Padova, vol. 124, pp. 231-246, 2010.

[21] L. Miao, "Finite groups with some maximal subgroups of Sylow subgroups $Q$-supplemented," Commun. Algebra, vol. 35, no. 1, pp. 103-113, 2007.

[22] M. Ramadan, "Influence of normality on maximal subgroups of Sylow subgroups of a finite group," Acta Math. Hung., vol. 59, no. 1-2, pp. 107-110, 1992.

[23] M. Ramadan, M. E. Mohamed, and A. A. Heliel, "On $c$-normality of certain subgroups of prime power order of finite groups,” Arch. Math., vol. 85, no. 3, pp. 203-210, 2005.

[24] D. J. S. Robinson, A course in theory of group. Berlin: Springer-Verlag, 1982.

[25] A. N. Skiba, "On weakly $s$-permutable subgroups of finite groups," J. Algebra, vol. 315, no. 1, pp. 192-209, 2007.

[26] A. N. Skiba, "On two questions of L. A. Shemetkov concerning hypercyclically embedded subgroups of finite groups," J. Group Theory, vol. 13, no. 6, pp. 841-850, 2010.

[27] A. N. Skiba, "A characterization of the hypercyclically embedded subgroups of finite groups," $J$. Pure Appl. Algebra, vol. 215, no. 3, pp. 257-261, 2011.

[28] Y. Wang, H. Wei, and Y. Li, "A generalization of Kramer's theorem and its application," Bull. Austral. Math. Soc., vol. 65, no. 3, pp. 467-475, 2002.

[29] Y. Wang, "C -normality of groups and its properties," J. Algebra, vol. 180, no. 3, pp. 954-965, art. no. $0103,1996$.

[30] H. Wei, "On $c$-normal maximal and minimal subgroups of Sylow subgroups of finite groups," Commun. Algebra, vol. 29, no. 5, pp. 2193-2200, 2001.

[31] H. Wei, Y. Wang, and Y. Li, "On $c$-normal maximal and minimal subgroups of Sylow subgroups of finite groups. II,” Commun. Algebra, vol. 31, no. 10, pp. 4807-4816, 2003.

[32] H. Wei, Y. Wang, and Y. Li, "On $c$-supplemented maximal and minimal subgroups of Sylow subgroups of finite groups," Proc. Am. Math. Soc., vol. 132, no. 8, pp. 2197-2204, 2004.

[33] M. Weinstein, Ed., Between nilpotent and solvable. Passaic, New Jersey (USA): Polygonal Publishing House, 1982.

[34] Q. Zhang and L. Wang, "The influence of $s$-semipermutable subgroups on the structure of finite groups," Acta Math. Sin., vol. 48, no. 1, pp. 81-88, 2005.

[35] X. Zhong and S. Li, "On $c$-supplemented minimal subgroups of finite groups," Southeast Asian Bull. Math., vol. 28, no. 6, pp. 1141-1148, 2004.

\section{Authors' addresses}

\section{Changwen Li}

School of Mathematics and Statistics, Jiangsu Normal University, Xuzhou, 221116, China

E-mail address: 1cw2000@126.com

\section{Xuemei Zhang}

Department of Basic Sciences, Yancheng Institute of Technology, Yancheng, 224051, China

E-mail address: xmzhang807@sohu.com

\section{Xiaolan Yi}

School of Science, Zhejiang Sci-Tech University, Hanzhou, 310018, China

E-mail address: yixiaolan2005@126.com 